\title{
Mode-Locking Dynamics in Electrically Driven Vertical-External-Cavity Surface-Emitting Lasers
}

\author{
Josep Mulet and Salvador Balle, Member, IEEE
}

\begin{abstract}
We develop a novel description of electrically driven vertical-external-cavity surface-emitting semiconductor lasers (VECSELs) mode-locked by saturable absorber mirrors. Our approach is based on an analytical solution of the bidirectional traveling-wave equations for fundamental transverse mode operation. The resulting time-domain equations describe the evolution of the electric fields and carrier-densities at the quantum-well layers of the emitter and absorber structures which are coupled through delayed boundary conditions. For the design considered, we obtain stable mode-locked pulses of few tens of picoseconds at $15-\mathrm{GHz}$ repetition rate in agreement with recently reported experimental results.
\end{abstract}

Index Terms-Mode-locked lasers, semiconductor device modeling, semiconductor lasers, surface-emitting lasers.

\section{INTRODUCTION}

$\mathbf{V}$ ERTICAL-EXTERNAL-CAVITY surface-emitting semiconductor lasers (VECSELs) are an interesting alternative to edge-emitters in high-power applications when fundamental transverse mode performance is required [1]. They are also suitable for intracavity frequency conversion, gain-switching and mode-locking applications. The external-cavity offers interesting features for mode-locking: good beam quality, possibility of laser mode control, and repetition rates up to tens of gigahertz. The initial demonstrations of mode-locked VECSELs used optically pumped devices, and active mode-locking was achieved via synchronous pumping [2] while passive mode-locking was obtained with semiconductor saturable absorber mirrors (SESAM) [3]. These devices generated picosecond pulses with output powers larger than $200 \mathrm{~mW}$ [4], [5]. Later studies demonstrated that sub-ps pulses can be obtained when the VECSEL operates in the so-called quasi-soliton mode-locking regime [6], [7]—an adequate amount of cavity dispersion compensates for unbalanced chirp in the amplifier and the saturable absorber. The greatest average optical power reported to date is $\sim 1 \mathrm{~W}$, and it seems that the electrical and thermal engineering of the device are the key to an improved performance [8]. The development of electrically driven VECSELs is challenging, in part, due to the unavoidable optical losses and heating generated in the doped layers. Notwithstanding, several demonstrations of electrically

Manuscript received April 6, 2005; revised May 27, 2005. This work was supported in part by the Project TIC2002-04255-C04-01 and COST288 Action "Nanoscale and Ultrafast Photonics." The work of J. Mulet was also supported in part by the Consejo Superior de Investigaciones Científicas through the Program I3P-2003.

The authors are with the Institut Mediterrani d'Estudis Avançats, IMEDEA (CSIC-UIB), Campus Universitat de les Illes Balears, E-07122 Palma de Mallorca, Spain (e-mail: mulet@imedea.uib.es).

Digital Object Identifier 10.1109/JQE.2005.853355 driven VECSELs lasing at wavelengths $\sim 1 \mu \mathrm{m}$ [9]-[11] and $\sim 1.55 \mu \mathrm{m}$ [12] have recently been reported. Jasim et al. [11] considered two distant vertical cavities, an emitter and a saturable absorber, coupled in a linear resonator. A reverse biased saturable absorber provided fast recovery of the absorption down to few picoseconds. Jasim et al. found mode-locked pulses of few tens of picoseconds at repetition rates up to $15 \mathrm{GHz}$. These experimental results motivated us to develop a detailed theoretical description in view of the optimization of the devices.

The regeneration of a signal in a vertical cavity device simultaneously involves amplification/absorption and filtering due to the high finesse of the Fabry-Pérot cavities defined by the distributed Bragg reflectors (DBRs). A correct description of these processes is essential since it determines the saturation properties of the device and defines the attainable pulsewidth. Different theoretical frameworks have been used to analyze passive mode-locking in VECSELs: 1) Haus master equation [13]; 2) numerical iterative models [6], [14]; and 3) a fully distributed time-domain model [15]. The classical viewpoint in Haus master equation is that of a pulse circulating in the resonator defined by the two vertical cavities. The pulse experiences regeneration when passing through the emitter and absorber cavities; dispersion and nonlinearity are treated separately and lumped into characteristic operators. The numerical iterative models follow the master equation but they incorporate in succession the effects encountered by the pulse, so the weak saturation approximation can be avoided. On the other side, a fully distributed time-domain model for extended-cavity VCSELs has recently been developed [15]. This model provides a detailed description of the propagation of the intracavity electric fields (within the vertical cavities) and the interaction with the active quantum wells (QWs). Thereby, the intracavity electric field directly experiences the effect of nonlinear gain saturation and dispersion while multiple pass regeneration accounts for bandwidth filtering. Therefore, no $a$ priori assumptions on either the filtering function or dispersion are required since they naturally arise from the user-defined optical structure. The major drawback, however, is the larger computational requirements associated with the spatial discretization along the cavity axis.

In this paper, we develop an analytical approximation to the bidirectional traveling-wave equations that allows us to alleviate the complexity of the distributed time-domain models. Our approach is justified in VECSELs since the light-matter response is linear everywhere except at the several QW layers distributed along the cavity. Therefore, the electric fields can be analytically propagated in the different linear layers, e.g., using the scattering matrix formalism, whereas the interaction with 


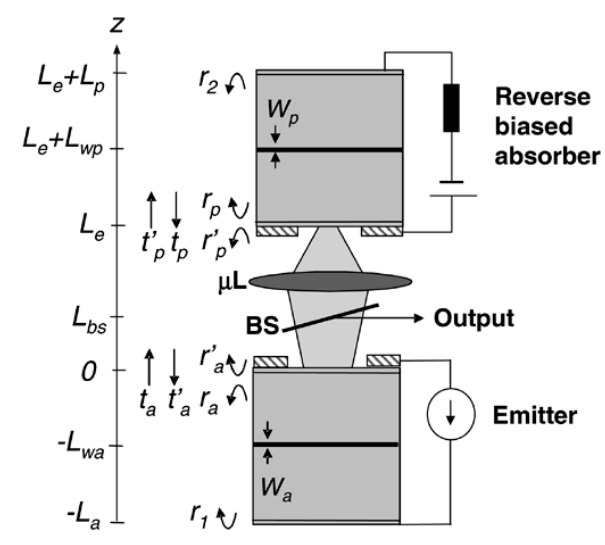

Fig. 1. Sketch of a VECSEL (not in scale). For clarity, the DBRs are represented as plane mirrors with effective internal (external) reflectivities $r$ $\left(r^{\prime}\right)$ and transmissivities $t\left(t^{\prime}\right)$. Beam splitter (BS) and microlens $(\mu \mathrm{L})$.

the thin QWs can be reduced to ordinary evolution equations. The paper is organized as follows. In Section II, the details of the VECSEL structure under consideration are specified. Section III presents the physical model for describing the interaction of a monochromatic optical field with the emitter and absorber cavities. In Section IV, we derive evolution equations in the time-domain by invoking the slowly varying approximation. Numerical simulations of the time-domain model are reported in Section V. For operation close to the laser threshold, we obtain stable mode-locked pulses of few tens of picoseconds at $15-\mathrm{GHz}$ repetition rate in agreement with experimental findings [11]. The mechanisms that determine the onset of mode-locking and the pulsewidth are discussed. Finally, Section VI is devoted to concluding the paper and to outline potential applications of our model.

\section{LASER STRUCTURE}

The mode-locked laser under study is sketched in Fig. 1. The structure of the VECSEL is similar to that of the device used in the experiments of [11]. It consists of two similar verticalcavity structures (emitter and absorber) a distance $L_{e}$ apart. The repetition rate is selected by adjusting the external-cavity length $L_{e}$.

The emitter contains a multiple quantum well (MQW) gain structure embedded in a $m-\lambda$ cavity defined by two distributed Bragg reflectors. The bottom, p-type DBR has high reflectivity ( $R \sim 0.997)$ while the top, n-type DBR has only moderate reflectivity, $R \sim 0.7-0.8$, and it incorporates a ring-contact that allows for electrical injection. The moderate reflectivity of the top DBR increases the optical losses in the emitter up to the point that it cannot lase by itself unless it receives feedback from the external saturable absorber mirror. Hence, it acts as a Fabry-Pérot optical amplifier where the larger the reflectivity the larger the amplified gain at the cost of reducing the available bandwidth.

The saturable absorber has a single quantum well (SQW) active region in order to reduce the unsaturated losses. The cavity is reverse biased to provide fast recovery of the absorption. Moreover, the reflectivity of the top mirror is approximately that of a semiconductor-air interface $(R \sim 0.3)$.
Both emitter and absorber are optically coupled through a microlens and a beam splitter with transmissivity $t_{\mathrm{bs}}$, which is also used as output coupler. The angled beam splitter introduces different losses for the TE and TM modes, hence, helping to select a linear polarization for the electric field.

\section{PHYSICAL MODEL}

In order to derive an optical model for the VECSEL, we start from the wave equation for a monochromatic wave expressed in the frequency domain. The propagation equation for the fundamental transverse mode is solved in the linear layers, and the boundary conditions that define the three coupled cavities - the emitter, the absorber and the external cavity_are imposed.

\section{A. Optical Model}

As depicted in Fig. 1, our system comprises the two vertical cavities plus the external cavity. Thus, the profile of each transverse mode varies along the cavity. Within the vertical cavities, the wave equation for a linearly polarized electric field $\mathcal{E}_{\omega}(\vec{r})$ in the frequency domain reads

$$
\nabla^{2} \mathcal{E}_{\omega}(\vec{r})+\frac{\omega^{2}}{c^{2}} n_{s}^{2}\left(\vec{r}_{\perp}\right) \mathcal{E}_{\omega}(\vec{r})=-\frac{\omega^{2}}{c^{2} \varepsilon_{0}} \mathcal{P}_{\mathrm{qW}}(\vec{r}, \omega)
$$

where $n_{s}^{2}\left(\vec{r}_{\perp}\right)=1+\chi_{s}$ stands for the distribution of the background refractive index of the GaAs spacers, and $\mathcal{P}_{\mathrm{qw}}(\vec{r}, t)$ the distribution of material polarization from the QWs. The lateral distribution of $n_{s}\left(\vec{r}_{\perp}\right)$ arises from the temperature dependence of the index of refraction and possible oxide apertures.

The field profiles in both vertical cavities are mutually dependent through propagation in the external cavity. In the external cavity, the paraxial optical beams can be propagated using, for instance, the Huygens' integral in the Fresnel approximation [16]. Following this procedure it is possible to determine the fundamental transverse mode $\Phi_{\omega}\left(r_{\perp} ; z\right)$ everywhere in the compound cavity. We assume that the microlens has a stronger effect defining the spatial mode than the refractive index distribution inside the vertical cavities. Hence, the fundamental transverse mode will be approximated by a paraxial Gaussian beam and weak spatial mode distortions, due to dynamical Kerr and thermal lensing effects, will be disregarded.

We consider fundamental transverse-mode operation of the VECSEL. We recall that this is the usual operation condition obtained experimentally after a proper design of the resonator. Within each vertical cavity, the electric field is expressed as a longitudinal profile times the fundamental transverse mode

$$
\mathcal{E}_{\omega}(\vec{r})=E_{\omega}(z) \Phi_{\omega}\left(\vec{r}_{\perp}\right)
$$

We have assumed that the mode $\Phi_{\omega}\left(\vec{r}_{\perp}\right)$ varies little while propagating within the vertical cavity. By using the effective-index approximation, the equation for $\Phi_{\omega}\left(\vec{r}_{\perp}\right)$ reads

$$
\nabla_{\perp}^{2} \Phi_{\omega}\left(\vec{r}_{\perp}\right)+\frac{\omega^{2}}{c^{2}} n_{s}^{2}\left(\vec{r}_{\perp}\right) \Phi_{\omega}\left(\vec{r}_{\perp}\right)=\beta^{2} \Phi_{\omega}\left(\vec{r}_{\perp}\right)
$$

with $\beta \equiv(\omega / c) n_{e}$, and $n_{e}$ being the modal refractive index. 
Once the transverse profile is determined for the VECSEL structure in Fig. 1, the optical problem is reduced to the longitudinal propagation of the optical field. The active region of each vertical cavity has a QW structure. In the general case, the QWs are distributed in several antinodes of the standing wave pattern of the $m-\lambda$ cavity. In order to simplify the description, we assume that all the wells are identical and in the same electronic state, so that the MQW structure is replaced by a SQW structure with a relative confinement factor scaled by the number of wells. In the limit that the QW thickness is much smaller than the wavelength, $W \ll \lambda$, the distribution of material polarization can be approximated by a Dirac's delta

$$
\begin{aligned}
\mathcal{P}_{\mathrm{qw}}(\vec{r}, \omega) & =\varepsilon_{0} \Delta \chi(\omega, N) \mathcal{E}_{\omega}\left(\vec{r}_{\perp}, z_{\mathrm{qw}}\right) W \delta\left(z-z_{\mathrm{qw}}\right) \\
\Delta \chi & \equiv\left[\chi^{n l}\left(\omega, N\left(\vec{r}_{\perp}\right)\right)+\chi_{\mathrm{qw}}\left(\vec{r}_{\perp}\right)-\chi_{s}\left(\vec{r}_{\perp}\right)\right]
\end{aligned}
$$

with $\chi^{n l}\left(\omega, N\left(\vec{r}_{\perp}\right)\right)$ the optical susceptibility of the active QW layer at a carrier density $N\left(\vec{r}_{\perp}\right), \chi_{\mathrm{qw}}$ the background contribution to the refractive index of InGaAs, $W$ the QW thickness, and $z_{\mathrm{qw}}$ the position of the QW in the cavity.

In this limit, the longitudinal field $E_{\omega}(z)$ obeys

$$
\begin{aligned}
& d_{z}^{2} E_{\omega}(z)+\frac{\omega^{2}}{c^{2}} n_{e}^{2} E_{\omega}(z)=-\frac{\omega^{2}}{c^{2}} \overline{\Delta \chi} E_{\omega}(z) W \delta\left(z-z_{\mathrm{qw}}\right) \\
& \overline{\Delta \chi}=\iint \Delta \chi^{n l}\left(\omega, N\left(\vec{r}_{\perp}\right)\right)\left|\Phi_{\omega}\left(\vec{r}_{\perp}\right)\right|^{2} d^{2} \vec{r}_{\perp}
\end{aligned}
$$

where $\overline{\Delta \chi}$ stands for the modal optical susceptibility.

The problem thus becomes linear everywhere in the optical cavity except for the interaction with the QW layers. In the limit of thin QWs, these interaction terms appear only as boundary conditions, since (5) imposes the continuity of the electric field $E_{\omega}(z)$ at every point in space with a discontinuity of its longitudinal derivative at each QW plane

$$
d_{z} E_{\omega}\left(z_{\mathrm{qw}}^{+}\right)-d_{z} E_{\omega}\left(z_{\mathrm{qw}}^{-}\right)=-\frac{\omega^{2}}{c^{2}} W \overline{\Delta \chi} E_{\omega}\left(z_{\mathrm{qw}}\right) .
$$

We solve the longitudinal propagation by expressing the axial dependence of the electric field as a set of counter-propagating waves

$$
\begin{aligned}
& E_{\omega}(z) \\
& \quad= \begin{cases}L^{+} e^{i q z}+L^{-} e^{-i q z}, & -L_{a} \leq z \leq-L_{w a} \\
A^{+} e^{i q z}+A^{-} e^{-i q z}, & -L_{w a}<z \leq 0 \\
C_{L}^{+} e^{i q_{o} z}+C_{L}^{-} e^{-i q_{o} z}, & 0<z \leq L_{\mathrm{bs}} \\
C_{R}^{+} e^{i q_{o} z}+C_{R}^{-} e^{-i q_{o} z}, & L_{\mathrm{bs}}<z \leq L_{e} \\
P^{+} e^{i k z}+P^{-} e^{-i k z}, & L_{e}<z \leq L_{e}+L_{w p} \\
R^{+} e^{i k z}+R^{-} e^{-i k z}, & L_{e}+L_{w p}<z \leq L_{e}+L_{p}\end{cases}
\end{aligned}
$$

with $q=(\omega / c) n_{a}(\omega), k=(\omega / c) n_{p}(\omega)$, and $n_{a}, n_{p}$ the (modal) refractive indexes of the emitter and absorber, respectively. The wells are located at $z=-L_{w a}$ and $z=L_{e}+L_{w p}$ in the cavities. Within the external cavity, we take into account localized loss at the position of the beam splitter with transmissivity $t_{\mathrm{bs}}$. Distributed losses due to other possible sources are modeled as $q_{0}=\omega / c+i \gamma$, that results in a one-way field attenuation $\zeta \equiv \exp \left(-\gamma L_{e}\right)$. The different plane waves in
(8) must verify adequate boundary conditions. At the position of the QWs, the electric field has to be continuous and (7) must be imposed. The reflectivity $r_{x}$ and transmissivity $t_{x}$ of the distributed Bragg reflectors impose the remaining boundary conditions. Their spectra are obtained from the classical transfer matrix method [17]. Primed symbols are used to indicate external reflectivity and transmissivity, i.e., when a field is injected from the external cavity to a vertical cavity.

The next step is to write the aforementioned boundary conditions in a more convenient form. At this point, it is worth noting that the nonlinear interactions involve the electric fields at the QW layers $E_{\mathrm{qw} A}$ and $E_{\mathrm{qw} P}$ defined in (8) for $z=-L_{w a}$ and $z=L_{e}+L_{w p}$, respectively. After a tedious although straightforward algebra, the boundary conditions at the emitter can be written in the form

$$
\begin{aligned}
\left(1-r_{1} r_{a} e^{i 2 q L_{a}}\right) E_{\mathrm{qw} A} & =i c_{A} \Gamma_{A} \overline{\Delta \chi_{A}}\left(\omega, N_{A}\right) E_{\mathrm{qw} A}+Y_{A} \\
Y_{A} & \equiv t_{a}^{\prime} e^{i q L_{w a}}\left(1+\tilde{r}_{1}\right) C_{L}^{-}
\end{aligned}
$$

with $c_{x}=(\omega / c) M_{x} W_{x} / n_{x}$, and $M_{x}$ the number of QWs. Equation (9) only involves the electric field at the QW layer and the externally injected field $Y_{A}$ acting onto the QW plane. The term on the left-hand side of this equation is the usual Fabry-Pérot function that describes multiple pass regeneration and introduces bandwidth filtering. The different parameters are $\tilde{r}_{1}=r_{1} \exp \left(i 2 q\left(L_{a}-L_{w a}\right)\right), \tilde{r}_{a}=r_{a} \exp \left(i 2 q L_{w a}\right)$, and the relative confinement factor $\Gamma_{A}=(1 / 2)\left(1+\tilde{r}_{1}\right)\left(1+\tilde{r}_{a}\right)$. Close to resonance $\left|\Gamma_{A}\right|$ can be as large as two [18]. Similarly for the absorber, we arrive at

$$
\begin{aligned}
\left(1-r_{2} r_{p} e^{i 2 k L_{p}}\right) E_{\mathrm{qw} P} & =i c_{P} \Gamma_{P} \overline{\Delta \chi_{P}}\left(\omega, N_{P}\right) E_{\mathrm{qw} P}+Y_{P} \\
Y_{P} & \equiv t_{p}^{\prime} e^{i k L_{w p}}\left(1+\tilde{r}_{2}\right) \zeta e^{i \frac{\omega}{c} L_{e}} C_{R}^{+}
\end{aligned}
$$

For convenience, $Y_{P}$ is defined as the injection field referenced at the absorber QW plane. The other parameters read $\tilde{r}_{2}=$ $r_{2} \exp \left(i 2 k\left(L_{p}-L_{w p}\right)\right), \tilde{r}_{p}=r_{p} \exp \left(i 2 k L_{w p}\right)$, and $\Gamma_{P}=$ $(1 / 2)\left(1+\tilde{r}_{2}\right)\left(1+\tilde{r}_{p}\right)$.

Equations (9)-(11) are the starting point of our optical model. They describe the fields in two Fabry-Pérot cavities which experience optical injection. In our case, however, the injection fields are not independent but connected through multiple pass propagation in the external cavity. The injected fields are expressible as

$$
\begin{aligned}
& Y_{A}(\omega)=t_{\mathrm{bs}} \zeta e^{i \frac{\omega}{c} L_{e}}\left[a_{1}(\omega) Y_{P}(\omega)+a_{2}(\omega) E_{\mathrm{qw} P}(\omega)\right] \\
& Y_{P}(\omega)=t_{\mathrm{bs} \zeta} \zeta e^{i \frac{\omega}{c} L_{e}}\left[p_{1}(\omega) Y_{A}(\omega)+p_{2}(\omega) E_{\mathrm{qw} A}(\omega)\right]
\end{aligned}
$$

where $a_{1,2}(\omega)$ and $p_{1,2}(\omega)$ are given in Appendix I. These equations could be used to eliminate the $Y_{A, P}$ fields in (9)-(11). However, we prefer not to do so since, in the present form, there is a clear separation between internal and external time scales that solely appear in the $\exp \left(i(\omega / c) L_{e}\right)$ terms of (13)-(14). This will be a key point when deriving the time-domain equations in Section IV. 


\section{B. Material Model}

As found experimentally, the typical duration of the pulses emitted by the electrically driven VECSEL is of the order of tens of ps. In this limit, the material polarization can be safely adiabatically eliminated and effectively described by an optical susceptibility. The imaginary part of this function describes the absorption spectrum and the real part contains the carrier-induced refractive index spectrum, that is generally found invoking the Kramers-Krönig relations.

Ultrafast processes, such as, spectral hole-burning (SHB) and carrier heating, modify the saturation properties of these devices [19]. Recently, it has been demonstrated that the effect of SHB can be analytically described through a field-dependent broadening of the optical transitions, which results in compression of both the gain and carrier-induced refractive index [20]. This analytical approximation allows for an efficient description of the nonlinear gain saturation and self-phase modulation effects at time scales above one picosecond.

In this paper, we use the analytical expressions for the full optical susceptibility (gain and refractive index) given in [20] and [21]

$$
\begin{aligned}
\chi_{A}^{n l}\left(\omega, D_{A}, E_{\mathrm{qw} A}\right)= & \frac{\Lambda_{A}+1}{2 \Lambda_{A}} f_{A}+\frac{\Lambda_{A}-1}{2 \Lambda_{A}} f_{A}^{*} \\
f_{A}= & -\chi_{0 A}\left[2 \ln \left(1-\frac{D_{A}}{u_{A}+i \Lambda_{A}}\right)\right. \\
& \left.-\ln \left(1-\frac{b_{A}}{u_{A}+i \Lambda_{A}}\right)\right] \\
\Lambda_{A}= & \sqrt{1+\epsilon_{A}\left|E_{\mathrm{qw} A}\right|^{2}}
\end{aligned}
$$

with $\epsilon_{A}$ the gain compression coefficient, and the normalized frequency $u_{A}=\left(\omega-E_{g A} / \hbar\right) / \gamma_{2 A}+\sigma\left(D_{A}\right)^{1 / 3} \cdot \sigma$ phenomenologically describes bandgap renormalization with the carrier density that has been normalized to the transparency value $D_{A} \equiv N_{A} / N_{t A}$. Finally, $\gamma_{2 A}$ stands for the material polarization dephasing-rate. The value of $\chi_{0 A}$ is taken as a free parameter to achieve a material gain about $1500 \mathrm{~cm}^{-1}$ per well at $D_{A}=2$.

In the saturable absorber, the expressions for the optical susceptibility are equivalent to (15) except for $u_{P}=\left(\omega-E_{g P} / \hbar\right) / \gamma_{2 P}$. Due to the quantum confined Stark effect in InGaAs/GaAs QWs, the bandgap experiences a redshift of $\sim 5 \mathrm{meV}$ per volt in reverse bias [22].

A final point consists in determining the carrier densities in the wells. In order to find a simple and efficient description, the transverse spatial dependence is eliminated by assuming homogeneous saturation energies in regions defined by the mode areas. As already mentioned, this approximation is justified when the transverse profiles are mainly defined by the microlens and the influence of Kerr and thermal lensing effects is weak. The evolution equations for the spatially averaged carrier densities, $D_{x}=\bar{N}_{x} / N_{t x}$ reads [23]

$$
\begin{aligned}
& \gamma_{e}^{-1} \partial_{t} D_{A}=\mu-D_{A}^{2}+\frac{1}{2 i}\left[\mathcal{P}_{A}(t) E_{\mathrm{qw} A}^{*}(t)-\text { c.c. }\right] \\
& \gamma_{e}^{-1} \partial_{t} D_{P}=-\gamma_{\mathrm{abs}} D_{P}+s \frac{1}{2 i}\left[\mathcal{P}_{P}(t) E_{\mathrm{qw} P}^{*}(t)-\text { c.c. }\right]
\end{aligned}
$$

where the material polarization reads

$$
\mathcal{P}_{x}(t)=\frac{1}{2 \pi} \int d \omega \chi_{x}^{n l}\left(\omega, D_{x}\right) E_{\mathrm{qw} x}(\omega) e^{-i \omega t} .
$$

Note that the electric fields and carrier densities are expressed as dimensionless quantities and nonradiative recombination has been neglected. The meaning of the different parameters is $\gamma_{e} \equiv B_{\text {eff }} N_{t A}, B_{\text {eff }}$ is the effective spontaneous recombination constant [18], $\gamma_{\mathrm{abs}} \equiv 1 /\left(\gamma_{e} \tau_{\mathrm{abs}}\right)$, and $\tau_{\mathrm{abs}}$ the absorber recovery time obtained from fitting of the experimental data [24], $\tau_{\mathrm{abs}}\left(V_{\mathrm{abs}}\right)=21 \mathrm{ps} \cdot \exp \left(V_{\mathrm{abs}} / 2.125 \mathrm{~V}\right)$ for $V_{\mathrm{abs}} \in[-4,0] \mathrm{V}$. The factor $s$ that multiplies the stimulated recombination term in the absorber equation reads $s \equiv\left(n_{a} N_{t A} w_{A}^{2}\right) /\left(n_{p} N_{t P} w_{P}^{2}\right)$, with $w_{x}$ the $1 / e$-beam waists of the modes in the each cavity. A rough estimate of the spot-area ratio is obtained assuming that the optical system is such that the emitter and absorber facets are conjugated planes through the microlens. The scaled current injected into the emitter is $\mu \equiv I /\left(e M_{\mathrm{qw}} W_{A} A_{\perp} B_{\mathrm{rad}} N_{t A}^{2}\right), e$ the elemental charge, $M_{\mathrm{qw}} W_{A}$ the total thickness of QWs, and $I$ the total injected current. The details of the current distribution, that should be obtained by solving the electrical conduction equation [25], [26], only define the effective active area in the emitter $A_{\perp}$.

The structure must provide good current confinement around the symmetry axis in order to reduce the effective active area $A_{\perp}$ and, in turn, the laser threshold current. Current spreading reduces the gain difference among transverse modes. Moreover, moderate threshold currents prevent self-heating of the emitter. The average increase in temperature can be expressed as $\Delta T_{\mathrm{av}}=R_{\mathrm{th}} Q_{T}, R_{\mathrm{th}}(\mathrm{K} / \mathrm{W})$ being the thermal resistance [27], and $Q_{T}$ the total amount of heat dissipated in the emitter. Due to the temperature increase, the main resonance shifts toward the blue side of the gain spectrum, which causes thermal roll-over. In addition, unbalanced heating in the emitter and absorber provides different resonant wavelengths, even though the cavities were resonant by construction.

\section{TIME-Domain EQUATIONS}

The physical model developed in the previous section describes the interaction of a monochromatic electric field with the QW layers. In this section, a time-domain version of the physical model is derived in order to access the mode-locking dynamics. A widely used method is the modal expansion in terms of compound-cavity modes, as performed in frequency-domain theories of mode-locking [28], [29]. However, they may become unpractical when the number of modes is large, as in the present case. Instead, we shall look for an analytical approximation of the Fourier integrals to time-domain. This approach is justified because of the different time scales-the round-trip times in the vertical cavities and the repetition rate of the external resonator-are clearly separated.

We define the slowly varying envelope (SVE) of the electric fields with respect to the carrier frequency $\omega_{0}, E(t)=\hat{E}(t) \exp \left(-i \omega_{0} t\right)$. The evolution of the electric fields at the QW layers, (9)-(11), admits a formal solution in the time-domain that is obtained by replacing $\omega \rightarrow \omega_{0}+i \partial_{t}$, thus accounting for dispersion at all orders. In order to arrive to a more treatable description, we look for an approximation 
of the resulting operator. For sufficiently long optical pulses, the Fabry-Pérot functions and the optical susceptibility can be expanded in Taylor series around $\omega_{0}$.

After Fourier transforming back to time-domain, the SVE of the electric fields $\hat{E}_{\mathrm{qw} A, P}$ at the QW layers evolves according

$$
\begin{aligned}
& \kappa_{A} \tau_{r t, A} \partial_{t} \hat{E}_{\mathrm{qw} A}(t) \\
&=-\left(1-\kappa_{A}\right) \hat{E}_{\mathrm{qw} A}(t) \\
& \quad+i c_{A} \Gamma_{A 0} \Delta \chi_{A}\left(\omega_{0}, D_{A}(t)\right) \hat{E}_{\mathrm{qw} A}(t)+\hat{Y}_{A}(t) \\
& \kappa_{P} \tau_{r t, P} \partial_{t} \hat{E}_{\mathrm{qw} P}(t) \\
&=-\left(1-\kappa_{P}\right) \hat{E}_{\mathrm{qw} P}(t) \\
&+i c_{P} \Gamma_{P 0} \Delta \chi_{P}\left(\omega_{0}, D_{P}(t)\right) \hat{E}_{\mathrm{qw} P}(t)+\hat{Y}_{P}(t)
\end{aligned}
$$

with $\kappa_{A}=r_{10} r_{a 0} e^{i 2 q L_{a}}$, and $\kappa_{P}=r_{20} r_{p 0} e^{i 2 k L_{p}}$. All quantities are computed at the SVE frequency $\omega_{0}$. The (complex) internal round-trip delays are defined by

$$
\begin{aligned}
\tau_{r t, A} & \equiv\left(\tau_{r_{1}}+\tau_{r_{a}}+\frac{2 L_{a}}{v_{g a}}\right)+\frac{c_{A}}{\kappa_{A}} \frac{\partial}{\partial \omega}\left[\Gamma_{A} \Delta \chi_{A}\right]_{\omega_{0}} \\
\tau_{r t, P} & \equiv\left(\tau_{r_{2}}+\tau_{r_{p}}+\frac{2 L_{p}}{v_{g p}}\right)+\frac{c_{P}}{\kappa_{P}} \frac{\partial}{\partial \omega}\left[\Gamma_{P} \Delta \chi_{P}\right]_{\omega_{0}}
\end{aligned}
$$

where $v_{g x}$ is the group velocity in the spacers, and $\tau_{r x}$ the reflection delays of the DBRs. It is worth noting that the last terms in the rhs of the above expressions describe, up to first order, gain and carrier-induced refractive index dispersion. More important $\tau_{r t, x}$ retain the dependence with the carrierdensities, hence, describing small timing adjustments through carrier-density fluctuations.

The structure of (19)-(20) is indeed very similar to the equations of two semiconductor lasers with mutual optical injection. The terms $\hat{Y}_{A}(t)$ and $\hat{Y}_{P}(t)$ represent the effective injection terms acting onto the $\mathrm{QW}$ layers. Their evolution is governed by the iterative equations

$$
\begin{aligned}
& \hat{Y}_{A}(t)=\xi\left[a_{1}\left(\omega_{0}\right) \hat{Y}_{P}\left(t-\tau_{e}\right)+a_{2}\left(\omega_{0}\right) \hat{E}_{\mathrm{qw} P}\left(t-\tau_{e}\right)\right] \\
& \hat{Y}_{P}(t)=\xi\left[p_{1}\left(\omega_{0}\right) \hat{Y}_{A}\left(t-\tau_{e}\right)+p_{2}\left(\omega_{0}\right) \hat{E}_{\mathrm{qw} A}\left(t-\tau_{e}\right)\right]
\end{aligned}
$$

that are obtained after Fourier transforming (13)-(14). As usual, hat denotes a slowly varying amplitude, $\xi=t_{\mathrm{bs}} \zeta \exp \left(i \omega_{0} L_{e} / c\right)$ is the one-way field attenuation, and the total one-way delay time $\tau_{e}=L_{e} / c+L_{w a} / v_{g, a}+L_{w p} / v_{g, p}+\tau_{t_{a}}+\tau_{t_{p}}$, with $\tau_{t_{x}}$ the transmission delays of the DBRs. The functions $a_{1,2}(\omega), p_{1,2}(\omega)$ are specified in Appendix I. Equations (23)-(24) define an iteration for the injection fields as a function of the delayed memory of the system valid for arbitrarily strong coupling. This effect can be easily numerically implemented by defining vectors that allocate the electric fields at the QWs and the injection fields. In this way, multiple reflections in the external cavity are naturally described.

The evolution of the carrier densities is governed by (16)-(17) except that the stimulated recombination terms are computed after a first-order expansion of the material polarization (18) around $\omega_{0}[23]$

$\hat{\mathcal{P}}_{x}(t) \approx \chi_{x}\left(\omega_{0}, D_{x}(t)\right) \hat{E}_{\mathrm{qw} x}(t)+i \partial_{\omega} \chi_{x}\left(\omega_{0}, D_{x}(t)\right) \partial_{t} \hat{E}_{\mathrm{qw} x}(t)$.

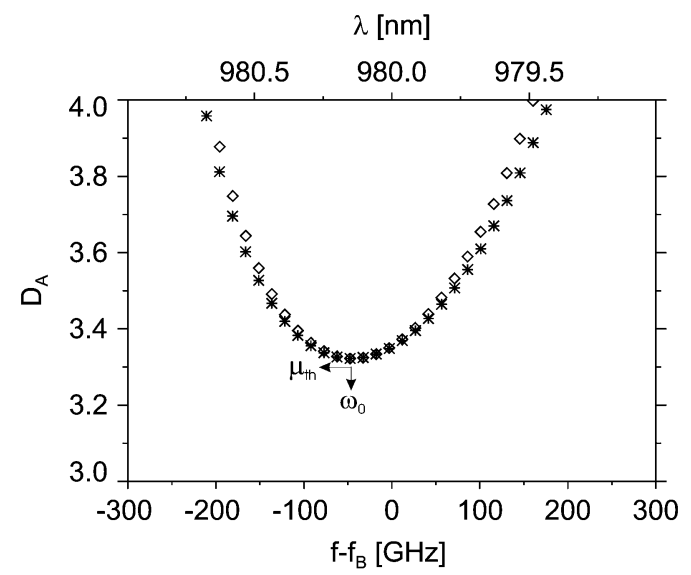

Fig. 2 .'Off"-state compound-cavity modes (inversion versus frequency) computed from the complete model $(\diamond)$ and the time-domain model $(*)$. The parameters are given in Section IV-A and $t_{\mathrm{bs}}=0.90$. The minima of these curves determine the laser threshold current $\mu_{\text {th }}$ and frequency $\omega_{0}$.

As a final remark, we would like to show that the time-domain model is a reasonable good approximation of the complete physical model. An easy way to understand the effect of the vertical cavities is to consider monochromatic injection fields. In this case, the solution of (19)-(20) has a Lorentzian lineshape whose modulus introduces filtering and its phase cavity dispersion. Hence, the Lorentzian lineshape approximates the characteristic Airy function of a Fabry-Pérot cavity around the carrier frequency, as a result of the truncated expansion in dispersion terms. Another test consists in comparing the compound-cavity modes at the "off"-state obtained from the complete and time-domain models. The monochromatic solutions at the "off" state verify the characteristic equation

$$
\begin{aligned}
& {\left[N_{11}(\omega)-i c_{A} \Gamma_{A} \Delta \chi_{A}\left(\omega, D_{A}\right)\right]} \\
& \quad \times\left[N_{22}(\omega)-i c_{P} \Gamma_{P} \Delta \chi_{P}(\omega, 0)\right]=N_{12}(\omega) N_{21}(\omega)
\end{aligned}
$$

that is a complex equation for two unknowns, $\omega$ and $D_{A}=$ $\sqrt{\mu}$. The coefficients of the matrix $N_{i j}(\omega)$ obtained from the complete physical model are given in Appendix II. The solutions of the characteristic equation are graphically shown in Fig. 2 for both the complete model (diamonds) and the time-domain model (stars). It can be seen that the modal frequencies and thresholds predicted by both models are in good agreement in a frequency interval $\sim 150 \mathrm{GHz}$ around the threshold mode; outside this interval, the expansion of the Fabry-Pérot functions becomes less accurate. The agreement between both models justifies the validity of the approximations involved in the time-domain model.

\section{A. Parameters}

The different parameter values used in the model are taken to reproduce the experimental conditions in [11]. For both the emitter and the absorber, the reference Bragg wavelength is $\lambda_{B}=980 \mathrm{~nm}$, while the reflectivity of bottom DBRs (23 pairs) is $R_{2}=R_{1}=0.998$. The reflectivity of the top DBR in the emitter is $R_{a}=0.75$ (4 pairs) while in the absorber we consider $R_{p}=0.30$ ( 1 pair). The modal refractive indexes are $n_{a}=n_{p}=3.52$, and the group refractive index is $n_{g}=4.5$. The QW thickness is $W_{A}=W_{P}=9 \mathrm{~nm}$, and the number of QWs $M_{\mathrm{qw} A}=4$ and $M_{\mathrm{qw} P}=1$. The susceptibility prefactor is 

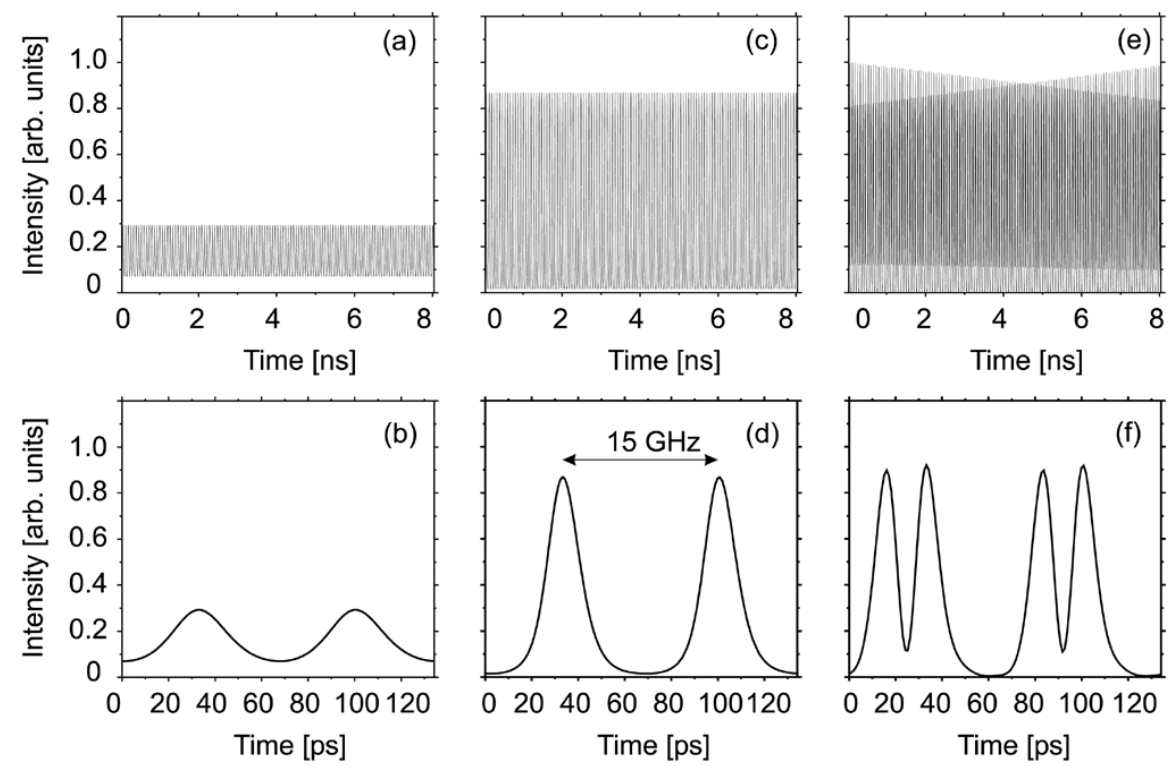

Fig. 3. Pulse train for different injection currents normalized to the laser threshold: (a)-(b) $\mu=1.2 \mu_{\text {th }}$; (c) - (d) $\mu=1.3 \mu_{\text {th }}$; and (e)-(f) $\mu=1.5 \mu_{\text {th. }}$. We go from oscillations, stable pulses, to incomplete mode-locking. Parameters are in Section IV-A except for $t_{\mathrm{bs}}=0.9$ and $s=8$.

$\chi_{0 A}=\chi_{0 P}=0.1$, the empty band contribution to the susceptibility in the emitter is $b_{A}=10^{4}$ and in the absorber $b_{P}=2 \cdot 10^{4}$. The gain suppression coefficient $\epsilon_{A}=\epsilon_{P}=10^{-23} \mathrm{~m}^{-3}$, the reference bandgap for the gain in the emitter $E_{g A}=1.265 \mathrm{eV}$ and in the absorber $E_{g P, \text { ref } f}=1.285 \mathrm{eV}$, the reverse bias in absorber $V_{\text {abs }}=-3 \mathrm{~V}$, the polarization dephasing rate in the emitter $\gamma_{2 A}=2 \cdot 10^{13} \mathrm{~s}^{-1}$ and in the absorber $\gamma_{2 P}=10^{13} \mathrm{~s}^{-1}$, the bandgap shrinkage coefficient in the emitter $\sigma=0.2$, the effective spontaneous recombination constant $B_{\text {eff }}=1.5 \cdot 10^{-16}$ $\mathrm{m}^{3} / \mathrm{s}$, the carrier density at transparency in the emitter $N_{t A}=$ $1.5 \cdot 10^{24} \mathrm{~m}^{-3}$ and in the absorber $N_{t P}=0.75 \cdot 10^{24} \mathrm{~m}^{-3}$. Finally, the effective active region area is $A_{\perp} \sim \pi \cdot 75^{2} \mu \mathrm{m}^{2}$, and the beam waist ratio $w_{a} / w_{p} \sim 2$. In order to operate close to the Bragg wavelength, the cavity lengths $L_{x}$ fulfill the usual resonance condition in a $m-\lambda$ cavity that reads

$$
n_{x}\left(L_{x}-M_{x} W_{x}\right)+n_{\mathrm{qw}, x} M_{x} W_{x}=m \lambda_{B}
$$

with $M_{x} W_{x}$ the total thickness of the QWs for $x=a, p$. The QW layers are placed in the center of the cavity close to an antinode of the standing wave pattern.

\section{NUMERICAL SIMULATIONS}

The time-domain model presented in the preceding section is defined as a system of nonlinear ordinary differential equations coupled to an algebraic delayed iteration for the injection fields. Tools for continuation and stability analysis of the mode-locked solutions are currently suitable for simpler delayed systems, e.g., as those describing two weakly coupled vertical cavities or a passively mode-locked ring laser [30]. This fact forces us to provide a numerical study of the mode-locked solutions.

\section{A. General Considerations}

In the emitter, the detuning between the Bragg and bandgap frequencies is set to $\Delta=\left(\omega_{B}-E_{g A} / \hbar\right) / \gamma_{2 A} \approx 1$ in order to operate close to the peak of maximum gain at typical carrier densities $D_{A} \approx 2-3$. Slightly red-shifted operation is beneficial due to the larger saturation fluence of the gain medium $E_{\mathrm{sat}, x}\left(\omega_{0}, N_{x}\right)=\pi w_{x}^{2} F_{\mathrm{sat}, x}, E_{\mathrm{sat}, x}$ being the (differential) saturation energy and $F_{\mathrm{sat}, x}$ the saturation fluence; although in this case there is an increase in threshold current. Alternatively, the ratio of saturation energies $E_{\mathrm{sat}, A} / E_{\mathrm{sat}, P}$ can be controlled by adjusting the aspect of mode beam waists $w_{x}$ within reasonable limits. A crucial condition to obtain stable mode-locking close to the laser threshold is the adjustment of the mode sizes and intensities of the standing-wave patterns in the emitter and the absorber. In particular, the mode has to be more tightly focused on the absorber facet in order to favor mode-locking. In the absorber, the nominal bandgap and the reverse bias are adjusted to obtain similar saturation fluences in the emitter and absorber. Hence, the operation in the absorber occurs around the absorption edge although not too far from the bandgap in order to have moderate absorption and positive alpha factors. The Henry alpha-factors, as function of the carrier frequency $\omega_{0}$ and the carrier density $N_{x}$, can be analytically obtained from the optical susceptibility (15), $\alpha_{x}\left(\omega_{0}, N_{x}\right)=\partial_{N} \operatorname{Re} \chi_{x}^{n l} / \partial_{N} \operatorname{Im} \chi_{x}^{n l}$. The typical values we obtain are $\alpha_{A} \approx 2-3$ in the emitter and $\alpha_{P} \approx 0-1$ in the absorber.

We start investigating the formation of the pulse train when the injection current in the emitter is gradually increased from the laser threshold.

\section{B. Scenario Upon Increase of the Injection Current}

Fig. 3 shows the pulse train for different injection levels in the emitter. The repetition rate is set to $15 \mathrm{GHz}$ as in the experiments reported in [11]. The initial transients that appear after each change in bias current have been removed. The general scenario is the following. When setting the bias current sufficiently close to the laser threshold, the VECSEL operates in 


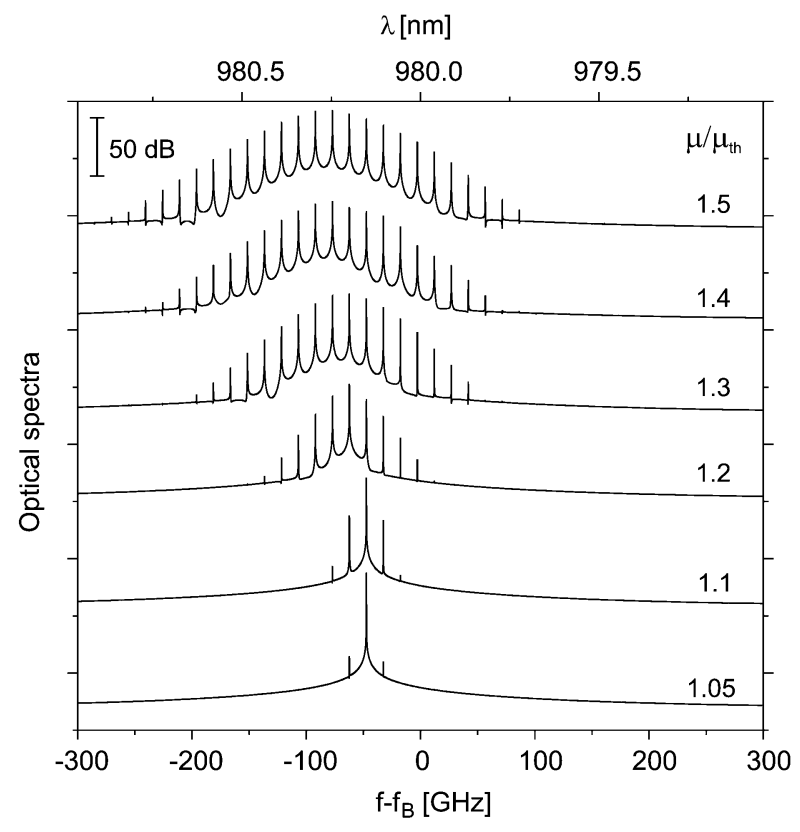

Fig. 4. Optical spectra for the same conditions described in Fig. 3. The plots have been vertically shifted for the sake of clarity.

a stable "on" compound-cavity-mode that corresponds to $\mathrm{cw}$ emission near the threshold mode $\omega_{0}$. Increasing the bias current, the output intensity undergoes harmonic oscillations at a frequency near the inverse of the cavity round-trip time. This fact is associated with the activation of longitudinal side modes. In fact, the optical spectrum for a bias current $\mu \approx 1.05 \mu_{\mathrm{th}}$ in Fig. 4, already displays the weak excitation of side modes of the main mode. This stage is followed by a rapid onset of multilongitudinal mode emission. A frequency comb, characteristic of mode-locking, develops in the different optical spectra. In the time-domain, the harmonic oscillations reshape into pulses when the current is increased. We consider the system to operate in a fully developed mode-locked regime when the suppression between the first and second harmonic peaks in the power spectrum is less than $3 \mathrm{~dB}$.

The dependence of the pulsewidth on the injection current is shown in Fig. 5. For well developed pulses, the pulsewidth is of the order of $10 \mathrm{ps}$ in agreement with experimental findings [11]. The pulses slightly shorten when the injection current increases. Nevertheless, there exists a maximum injection current accessible for fundamental transverse mode operation. The pulses are slightly chirped due to the combined effect of self-phase modulation effects and cavity dispersion, leading to a spectral broadening of the pulses. The time-bandwidth product computed for these pulses is $\sim 0.5$.

For certain injection currents beyond threshold, modelocking may enter into an irregular region as shown in Fig. 3(e) and (f). In simulations, this instability leads to pulse splitting and a slow (ns $\sim \mu \mathrm{s}$ ) modulation of the pulse train. Experimentally, there is evidence that the laser can display a similar unstable self-pulsation regime [11]. At this stage, the quantities characterizing the pulse, such as the pulse energy, become time dependent. The instability point can be moved to higher currents by increasing the reverse bias applied to the absorber.

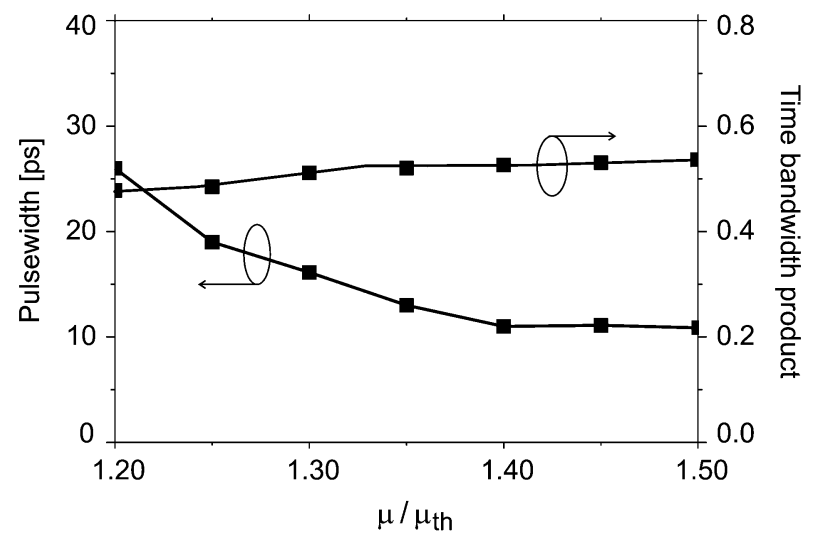

Fig. 5. Uncompressed pulsewidth and time bandwidth product as function of the injection current. The same parameters as in Fig. 3.

\section{CONCLUSION}

We have developed a novel time-domain description of passive mode-locking in electrically driven VECSELs. Our description analytically deals with the bidirectional traveling wave propagation in two coupled vertical cavities. In the time-domain, the model can be reduced to a set of algebraic differential equations with time delay that physically represents the delayed injection from one vertical cavity into its counterpart. The effects of dispersion and nonlinear saturation imposed by the vertical cavities are naturally accounted for by directly describing the evolution of the intracavity electric fields. In this sense, the description fills the gap between numerical iterative models and more sophisticated finite-difference theories. Our model enables a thorough understanding of the role played by the different design parameters on the mode-locking performance.

The results obtained from numerical simulations of the timedomain model show a good agreement with the experimental results reported recently by several groups. Stable mode-locked pulses of $\sim 10-$ ps temporal duration are obtained at $15-\mathrm{GHz}$ repetition rate. The optimization of the pulsewidth is an important issue in electrically driven devices that would require further investigation. The pulsewidth could be shortened by reducing the reflectivity of the top DBR in the emitter, the fact that also facilitates the mode-locking onset. However, there is a tradeoff between gain and bandwidth that has to be optimized in order to maintain the threshold characteristics of the device. We find that mode-locking starts near the laser threshold when the saturation energies in the emitter and absorber are adjusted through their saturation fluences, beam spot sizes, and standing wave intensities in the cavities. Thus, the effect of the mode coupling between the vertical cavities must be investigated in detail in order to identify the relevant design parameters defining the mode-locking onset.

Finally, we would like to mention other potential applications and extensions of the model. Since no a priori assumption on the solution is used, the formation of the pulse train can be analyzed as well as other general aspects of mode-locking, namely, noise characteristics, synchronization locking, etc. Transverse effects in the present model have been taken into account at a level that ensures accuracy and efficiency. The range of single transverse mode operation could be determined by allowing the oscillation 
of several transverse modes in the structure. For Gaussian mode operation, the influence of inhomogeneous gain saturation on the pulsewidth and pulse stability could be investigated by including the spatial hole-burning effects.

\section{APPENDIX I}

\section{ITERATION FUNCTIONS FOR THE INJECTION FIELDS}

The functions $a_{1,2}(\omega)$ and $p_{1,2}(\omega)$ define the iteration of the injection fields $Y_{A, P}$

$$
\begin{aligned}
& a_{1}(\omega)=\left(r_{p}^{\prime}-t_{p} t_{p}^{\prime} \frac{e^{i 2 k L_{w p}}}{1+\tilde{r}_{p}}\right) \frac{t_{a}^{\prime} e^{i q L_{w a}}\left(1+\tilde{r}_{1}\right)}{t_{p}^{\prime} e^{i k L_{w p}}\left(1+\tilde{r}_{2}\right)} \\
& a_{2}(\omega)=t_{p} t_{a}^{\prime} e^{i\left(q L_{w a}+k L_{w p}\right)} \frac{\left(1+\tilde{r}_{1}\right)}{\left(1+\tilde{r}_{p}\right)} \\
& p_{1}(\omega)=\left(r_{a}^{\prime}-t_{a} t_{a}^{\prime} \frac{e^{i 2 q L_{w a}}}{1+\tilde{r}_{a}}\right) \frac{t_{p}^{\prime} e^{i k L_{w p}}\left(1+\tilde{r}_{2}\right)}{t_{a}^{\prime} e^{i q L_{w a}}\left(1+\tilde{r}_{1}\right)} \\
& p_{2}(\omega)=t_{a} t_{p}^{\prime} e^{i\left(q L_{w a}+k L_{w p}\right)} \frac{\left(1+\tilde{r}_{2}\right)}{\left(1+\tilde{r}_{a}\right)} .
\end{aligned}
$$

The following notation is used: primed symbols stand for external reflectivity and transmissivity, $\tilde{r}_{1}=r_{1} \exp \left(i 2 q\left(L_{a}-\right.\right.$ $\left.\left.L_{w a}\right)\right), \tilde{r}_{a}=r_{a} \exp \left(i 2 q L_{w a}\right), \tilde{r}_{2}=r_{2} \exp \left(i 2 k\left(L_{p}-L_{w p}\right)\right)$, and $\tilde{r}_{p}=r_{p} \exp \left(i 2 k L_{w p}\right), q=(\omega / c) n_{a}$, and $k=(\omega / c) n_{p}$.

\section{APPENDIX II \\ COEFFicients OF THE $N_{i j}$ MATRIX}

$$
\begin{aligned}
N_{11} & =1-r_{1} r_{a} e^{i 2 q L_{a}}-\frac{\xi^{2}(\omega)}{\Delta} a_{1}(\omega) p_{2}(\omega) \\
N_{22} & =1-r_{2} r_{p} e^{i 2 k L_{p}}-\frac{\xi^{2}(\omega)}{\Delta} a_{2}(\omega) p_{1}(\omega) \\
N_{12} & =\frac{\xi}{\Delta} a_{2}(\omega), \quad N_{21}=\frac{\xi}{\Delta} p_{2}(\omega) \\
\Delta & =1-\xi^{2}(\omega) a_{1}(\omega) p_{1}(\omega)
\end{aligned}
$$

with $\xi(\omega) \equiv t_{\mathrm{bs}} \zeta e^{i(\omega / c) L_{e}}$ and $a_{1,2}$ and $p_{1,2}$ are the functions defined in Appendix I.

\section{ACKNOWLEDGMENT}

The authors would like to thank K. Yvind, T. Greibe, J. Mørk, and J. Javaloyes for fruitful discussions.

\section{REFERENCES}

[1] A. C. Tropper, H. D. Foreman, A. Garnache, K. G. Wilcox, and S. H. Hoogland, "Vertical-external-cavity semiconductor lasers," J. Phys. D: Appl. Phys., vol. 37, pp. R75-R85, 2004.

[2] W. Jiang, R. Mirin, and J. E. Bowers, "Mode-locked GaAs vertical cavity surface emitting lasers," Appl. Phys. Lett., vol. 60, pp. 677-679, 1992.

[3] U. Keller, K. J. Weingarten, F. X. Kärtner, D. Kopf, B. Braun, I. D. Jung, R. Fluck, C. Hönninger, N. Matuschek, and J. A. der Au, "Semiconductor saturable absorber mirrors (SESAMs) for femtosecond to nanosecond pulse generation in solid-state lasers," IEEE J. Sel. Topics Quantum Electron., vol. 2, no. 3, pp. 435-453, Sep. 1996.

[4] S. Hoogland, S. Dhanjal, A. C. Tropper, J. S. Roberts, R. Häring, R. Paschotta, F. Morier-Genoud, and U. Keller, "Passively mode-locked diode-pumped surface-emitting semiconductor lasers," IEEE Photon. Technol. Lett., vol. 12, no. 9, pp. 1135-1137, Sep. 2000.

[5] R. Häring, R. Paschotta, E. Gini, F. Morier-Genoud, D. Martin, H. Melchior, and U. Keller, "Picosecond surface-emitting semiconductor laser with $>200 \mathrm{~mW}$ average output power," Electron. Lett., vol. 37, pp. 766-768, 2001.
[6] R. Paschotta, R. Häring, A. Garnache, S. Hoogland, A. C. Tropper, and U. Keller, "Soliton-like pulse-shaping mechanism in passively modelocked surface-emitting semiconductor lasers," Appl. Phys. B, vol. 75, pp. $445-451,2002$.

[7] A. Garnache, S. Hoogland, A. C. Tropper, I. Sagnes, G. Saint-Girons, and J. S. Roberts, "Sub-500-fs soliton-like pulse in a passively modelocked broad-band surface-emitting laser with $100 \mathrm{~mW}$ average power," Appl. Phys. Lett., vol. 80, pp. 3892-3894, 2002.

[8] R. Häring, R. Paschotta, A. Aschwanden, E. Gini, F. Morier-Genoud, and U. Keller, "High-power passively mode-locked semiconductor lasers," IEEE J. Quantum Electron., vol. 38, no. 9, pp. 1268-1275, Sep. 2002.

[9] K. Jasim, Q. Zhang, A. V. Nurmikko, A. Mooradian, G. Carey, W. Ha, and E. Ippen, "Passively modelocked vertical extended cavity surface emitting diode laser," Electron. Lett., vol. 39, pp. 373-374, 2003.

[10] Q. Zhang, K. Jasim, A. V. Nurmikko, A. Mooradian, G. Carey, W. Ha, and E. Ippen, "Operation of a passively mode-locked extended-cavity surface-emitting diode laser in multiGhz regime," IEEE Photon Technol. Lett., vol. 16, no. 3, pp. 885-887, Mar. 2004.

[11] K. Jasim, Q. Zhang, A. V. Nurmikko, E. Ippen, A. Mooradian, G. Carey, and W. Ha, "Picosecond pulse generation from passively modelocked vertical cavity diode laser at up to $15 \mathrm{GHz}$ repetition rate," Electron Lett., vol. 40, pp. 34-36, 2004.

[12] M. El Kurdi, S. Bouchoule, A. Bousseksou, I. Sagnes, A. Plais, M. Strassner, A. Garnache, and J. Jacquet, "Room-temperature continuouswave laser operation of electrically pumped 1.55- $\mu \mathrm{m}$ VECSEL," Electron. Lett., vol. 40, pp. 671-672, 2004.

[13] H. A. Haus, "Theory of mode locking with a fast saturable absorber," $J$ Appl. Phys., vol. 46, pp. 3049-3058, 1975.

[14] O. E. Martinez, R. L. Fork, and J. P. Gordon, "Theory of passively mode locked-lasers for the case of a nonlinear complex-propagation coefficient," J. Opt. Soc. Amer. B, vol. 2, pp. 753-760, 1985.

[15] M. Bahl, H. Rao, N. C. Panoiu, and R. M. Osgood Jr., "Simulation of mode-locked surface-emitting lasers through a finite-difference time-domain algorithm," Opt. Lett., vol. 29, pp. 1689-1691, 2004.

[16] A. E. Siegman, Lasers. Mill Valley, CA: University science books, 1986.

[17] T. E. Sale, "Cavity and reflector design for vertical cavity surface emitting lasers," in IEE Proc. Optoelectron., vol. 142, 1995, pp. 37-43.

[18] S. F. Yu, Analysis and Design of Vertical Cavity Surface Emitting Lasers. New York: Wiley, 2003.

[19] A. Uskov, J. Mørk, and J. Mark, "Theory of short-pulse gain saturation in semiconductor laser amplifiers," IEEE Photon. Technol. Lett., vol. 4, no. 5, pp. 443-446, May 1992.

[20] S. Balle, "Analytical description of spectral hole-burning effects in active semiconductors," Opt. Lett., vol. 21, pp. 1923-1925, 2002.

[21] _ "Simple analytical approximations for the gain and refractive index spectra in quantum well lasers," Phys. Rev. A, vol. 57, pp. 1304-1312, 1998

[22] J. Hudgings, R. J. Stone, S. F. Lim, G. S. Li, W. Yuen, K. Y. Lau, and C. J. Chang-Hasnain, "The physics of negative differential resistance of an intracavity voltage-controlled absorber in a vertical-cavity surfaceemitting laser," Appl. Phys. Lett., vol. 73, pp. 1796-1798, 1998.

[23] J. Mulet and S. Balle, "Spatio-temporal optical model of VCSELs in the presence of polarization effects," IEEE J. Quantum Electron., vol. 38 no. 3, pp. 291-305, Mar. 2002.

[24] Q. Zhang, K. Jasim, A. V. Nurmikko, E. Ippen, A. Mooradian, G. Carey, and W. Ha, "Characteristics of a high-speed passively mode-locked surface-emitting semiconductor InGaAs laser diode," IEEE Photon. Technol. Lett., vol. 173, pp. 525-527, Mar. 2005.

[25] G. R. Hadley, K. L. Lear, M. E. Warren, K. D. Choquette, J. W. Scott, and S. W. Corzine, "Comprehensive numerical modeling of vertical-cavity surface emitting-lasers," IEEE J. Quantum Electron., vol. 32, no. 4, pp. 607-616, Apr. 1996

[26] M. Osinski, V. A. Smagley, M. Liu, G. A. Smolyakov, P. G. Eliseev, B. P Riely, P. H. Shen, and G. J. Simonis, "Self-consistent calculation of current self-distribution effect in GaAs-AlGaAs oxide-confined VCSELs," IEEE J. Sel. Topics Quantum Electron., vol. 9, no. 5, pp. 1422-1430, Sep./Oct. 2003.

[27] W. Nakwaski and M. Osinski, "Thermal properties of vertical-cavity surface-emitting lasers," in Progress in Optics XXXVIII, E. Wolf, Ed. Amsterdam, The Netherlands: Elsevier North-Holland, 1998, pp. $165-262$.

[28] J. F. Martins-Filho, E. A. Avrutin, C. N. Ironside, and J. S. Roberts, "Monolithic multiple colliding pulse mode-locked quantum-well lasers: Experiment and theory," IEEE J. Sel. Topics Quantum Electron., vol. 1, no. 2, pp. 539-551, Jun. 1995. 
[29] E. A. Avrutin, J. H. Marsh, and J. M. Arnold, "Modeling of semiconductor laser structures for passive harmonic mode locking at terahertz frequencies," Int. J. Optoelectron., vol. 10, pp. 427-432, 1995.

[30] A. G. Vladimirov, D. Turaev, and G. Kozyreff, "Delay differential equations for mode-locked semiconductor lasers," Opt. Lett., vol. 29, pp. 1221-1223, 2004.

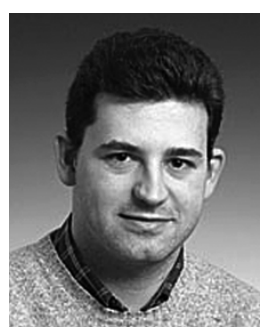

Josep Mulet was born in Lloseta, Mallorca, Spain, in 1975. He received the M.S. and Ph.D. degrees in physics from the Universitat de les Illes Balears, Palma de Mallorca, Spain, in 1998 and 2003, respectively.

During 2003, he was employed as Postdoctoral Researcher at the Research Center COM, Technical University of Denmark, Lyngby, Denmark. He is currently with the Institut Mediterrani d'Estudis Avançats, IMEDEA (CSIC-UIB), Campus Universitat de les Illes Balears, Palma de Mallorca, Spain. His research topics include mode-locking in extended-cavity semiconductor lasers, vertical-cavity surface-emitting semiconductor lasers and amplifiers, and synchronization of chaotic semiconductor lasers.

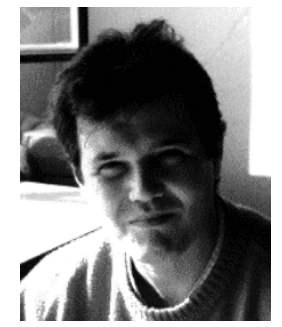

Salvador Balle (M'91) was born in Manacor, Mallorca, Spain in 1961. He received the Ph.D. degree in physics from the Universitat Autònoma de Barcelona, Barcelona, Spain in 1988.

$\mathrm{He}$ is currently with the Institut Mediterrani d'Estudis Avançats, IMEDEA (CSIC-UIB), Campus Universitat de les Illes Balears, Palma de Mallorca, Spain. His research interests are the nonlinear dynamics of semiconductor lasers and amplifiers and the modeling of the nonlinear optical properties of these devices. 